\title{
Optical Manifestations of Planar Chirality
}

\author{
A. Papakostas, ${ }^{1,2}$ A. Potts, ${ }^{1,2}$ D. M. Bagnall, ${ }^{1,2}$ S. L. Prosvirnin, ${ }^{3}$ H. J. Coles, ${ }^{2}$ and N. I. Zheludev ${ }^{1, *}$ \\ ${ }^{1}$ Department of Physics and Astronomy, University of Southampton, SO17 1BJ, United Kingdom \\ ${ }^{2}$ Department of Electronics and Computer Science, University of Southampton, SO17 1BJ, United Kingdom \\ ${ }^{3}$ Institute of Radio Astronomy of National Academy of Sciences of Ukraine, Kharkov, 61002, Ukraine
}

(Received 27 August 2002; published 14 March 2003)

\begin{abstract}
We report that planar chiral structures affect the polarization state of light in a manner similar to three-dimensional chiral (optical active) media. In experiments with artificial metal-on-silicon chiral planar gratings of 442 wallpaper group symmetry, containing millions of chiral elements per square centimeter, we observed rotation of the polarization azimuth in excess of $30^{\circ}$ of light diffracted from it. The rotation was found to change its sign for two enantiomeric forms of the media and to have components associated with both the structural arrangement and the chirality of individual structural elements.
\end{abstract}

DOI: $10.1103 /$ PhysRevLett.90.107404

The ability of left-right asymmetrical (chiral) threedimensional molecules to rotate the polarization state of light known as optical activity is one of the most remarkable effects in optics that has been extensively studied since its discovery at the beginning of the 19th century. An optical active medium (for example, a medium consisting of randomly oriented helixlike molecules) will show opposite signs of polarization azimuth rotation for the two mirror-symmetric (enantiomeric) forms of the constituting molecule. The general concept of chirality also exists in two dimensions [1-4], where a planar object is said to be chiral if it cannot be brought into congruence with its mirror image unless it is lifted from the plane. One could therefore envisage a planar chiral medium that consists of "flat" chiral elements possessing no line of symmetry in the plane. So far, there have been only a few theoretical publications on the optical manifestations of such planar chirality. Hecht and Barron predicted incoherent circular differential Rayleigh and Raman light scattering from an ensemble of planar chiral molecules [5]. They showed that genuine strong chiral scattering phenomena could be generated through pure electric dipole interactions (in comparison with the much weaker processes involving magneto-dipole interaction in threedimensional chirality), while Arnaut and Davis calculated the scattered fields from the two-dimensional chiral structure of a metallic wire gammadion and found rotation of the polarization azimuth of the scattered field [6]. However, there have as yet been no reports of experimental observations of any optical manifestations of planar chirality, apart from the observation of a random chiral component in a highly localized near-field polarization effect in metallic fractal aggregates [7]. Consequently, it has yet to be shown whether planar chiral media could affect the far-field polarization state of light scattered on it in a manner similar to three-dimensional chiral media when the polarization effect is sensitive to the handedness of the structure. Here we report that we have manufactured left- and right-handed forms of a regular artificial
PACS numbers: 78.67.-n, 78.20.Ek

medium consisting of microscopic chiral metallic objects distributed regularly in a plane, with a density of several millions per square centimeter. In this artificial medium we have observed handedness-sensitive rotations and elliptization of the polarization state for visible light diffracted from the structure.

We studied arrays of gammadions arranged in regular two-dimensional square gratings to produce a structure of planar 442 wallpaper group symmetry (in Orbifold notation, or " $\mathrm{p} 4$ " in International Union of Crystallography notation) [8]. This group has no axes of reflection or glide reflection and is $2 \mathrm{D}$ chiral with corresponding wallpaper structures available in two enantiomeric forms (see inset in Fig. 1). The structures were manufactured using a combination of direct-write electron beam lithography and ion beam milling on a thin film of metal $(100 \mathrm{~nm}$

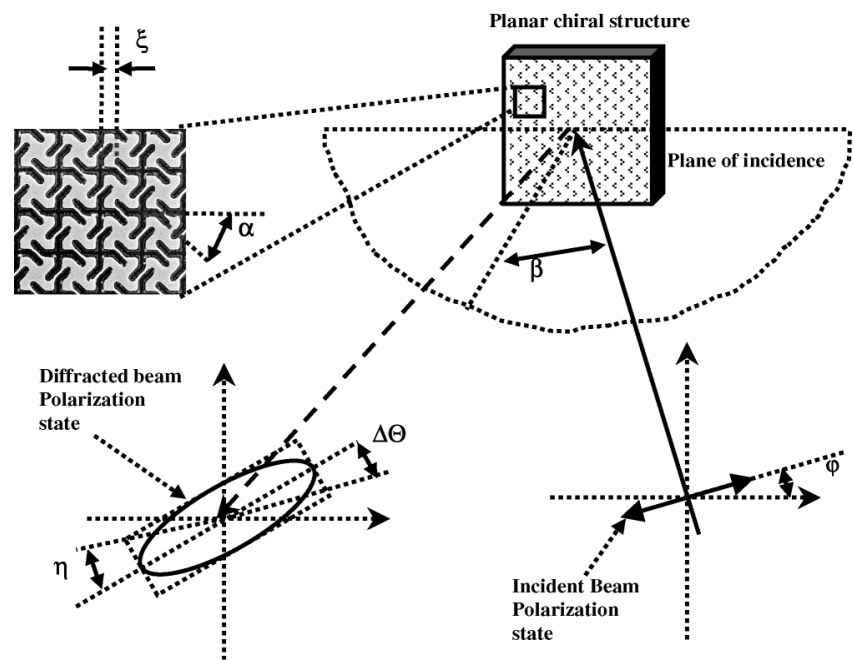

FIG. 1. A schematic illustration of the experiment to study optical manifestations of two-dimensional chirality in artificial planar structures. The inset shows a scanning electron micrograph of a typical chiral structure $(\mathrm{Ti} / \mathrm{Au} / \mathrm{Ti}$ on silicon). Dark areas correspond to removed metallic film. 
of gold sandwiched between two $20 \mathrm{~nm}$ thick layers of titanium) on a double-polished silicon substrate. The gammadions had sides of length $\xi=1.4 \mu \mathrm{m}$, but different internal angles $\alpha$, with positive values of $\alpha$ corresponding to clockwise gammadions and negative values corresponding to anticlockwise. The pitch of the gratings was $4.0 \mu \mathrm{m}$ in both directions so that the density of gammadions was $6.2 \times 10^{6} \mathrm{~cm}^{-2}$, while the total grating area available for optical measurements was approximately $1 \times 1 \mathrm{~mm}^{2}$. Optical experiments were performed at a wavelength of $632 \mathrm{~nm}$, and the polarization parameters of the diffracted wave were measured using the "rotating wave-plate" polarimetric technique. The structures were oriented to the incident beam in such a way that the plane of incidence was parallel to one axis of the gammadion array (see Fig. 1). In this configuration, diffracted waves propagate in both the plane of incidence and at various angles to it, with the scattered light showing a well-defined rectangular diffraction pattern. The polarization states of these diffracted waves are noticeably different from those which would be expected for diffraction from a nonchiral grating. If the diffracted wave remains in the plane of incidence and the incident wave is polarized either in the plane of incidence or perpendicular to the plane, no polarization change would be expected for a nonchiral grating. However, we found that if the incident wave is linearly polarized, say, in the plane of incidence, the polarization states of the diffracted waves are, in general, elliptical, with the polarization azimuth rotated by some angle to the initial polarization state. For a given angle of incidence $\beta$, the polarization states of various diffraction orders were found to be different. We concentrated on characterizing the polarization states of the first-order diffracted waves in the plane of incidence and measured the difference $\Delta \Theta$ between the polarization azimuth of the incident wave $\varphi$ and that of the diffracted wave. We also measured the ellipticity angle $\eta$ of the diffracted wave (where $\eta=0$ corresponds to a linearly polarized wave, and $\eta= \pm 45^{\circ}$ corresponds to right and left circular polarizations, accordingly [9]).

We first measured the diffracted beam polarization change as a function of the angle of incidence $\beta$ for three pairs of enantiomeric chiral structures with different gammadion internal angles $\alpha$, for an incident wave that was linearly polarized in the plane of incidence [see Fig. 2(a)]. The polarization azimuth rotation $\Delta \Theta$ and the degree of ellipticity $\eta$ both strongly depend on the angle of incidence $\beta$. However, whatever the values of $\beta$, $\Delta \Theta$, and $\eta$, the waves diffracted from enantiomeric planar chiral structures were of the same amplitude but of opposite sign. A comparison of results for the polarization azimuth rotation [Fig. 2(b)] for enantiomeric pairs with different values of $\alpha$ illustrates that the strongest polarization effects are seen in the "open" gammadion structures with $\alpha= \pm 45^{\circ}$. We also compared the polarization azimuth rotation for positive and negative incident angles, $\pm \beta$. Although some minor differences in the polarization response for incident angles of opposite signs were observed, the polarization changes were found to be largely insensitive to the sign of the incident angle. Our results [Figs. 2(a) and 2(b)] unambiguously show that polarization changes in light diffracted from a chiral planar structure show a true signature of planar chirality because the interaction of light with the two enantiomeric (left-right) forms of the structure yields different polarization changes that would not be observed if the structure lacked planar chirality. Comparison of the polarization changes resulting from light reflected from an unstructured gold surface with that for the two enantiomeric forms of the chiral structure for different incident polarizations [Fig. 2(c)] provides further evidence for true planar chirality. Incident waves linearly polarized with $\varphi=0^{\circ}$ and $90^{\circ}$ are eigenpolarizations of an unstructured metallic surface: the polarization azimuth and ellipticity of the waves do not change on reflection. By contrast, chiral planar structures exhibit strong polarization changes for both $\varphi=0^{\circ}$ and $90^{\circ}$ with the polarization changes having an opposite sign for each enantiomeric form in both cases. Moreover, the orientation mean values of both the polarization azimuth rotation and the ellipticity (each averaged over the incident polarization azimuth $\varphi$ ) are nonzero and have opposite sign, indicating that true planar chirality should also be observable for light scattered from randomly oriented gammadions. It should be noted that the geometry of the experiment imposes unequal conditions for the incident light polarized in the plane of incidence and perpendicular to the plane. As a result of this asymmetry, planar chirality appears on a background of "birefringence." In such a situation, one would expect the polarization change to be an alternating function of $\varphi$, as seen in this experiment. Such an alternating dependence has a clear analogy with the polarization change in the optical Faraday effect in a birefringent crystal. One can distinguish between two different hierarchy levels of planar chirality. The first is "molecular" chirality derived from the chirality of individual structural elements such as gammadions, which have no symmetry axes. Molecular chirality will survive even if structural elements are randomly oriented on the plane. The second source of planar chirality is due to the ordering of structural elements, which are not necessarily in themselves chiral. Here the chirality of the structure as a whole emerges as a result of ordering. Thus, for example, a square grid of crosses with equal sides would be chiral overall, and the structure will still have no axis of symmetry, if all the crosses are equally rotated by any angle with respect to the grid that is not a multiple of $45^{\circ}$, even though neither the crosses nor the grid are themselves chiral. Such planar "structural" chirality will, however, vanish if these nonchiral elements are randomly oriented in the plane. Analysis of Fig. 2(c) shows that the orientation mean value of the polarization azimuth rotation, which represents the molecular component of planar 

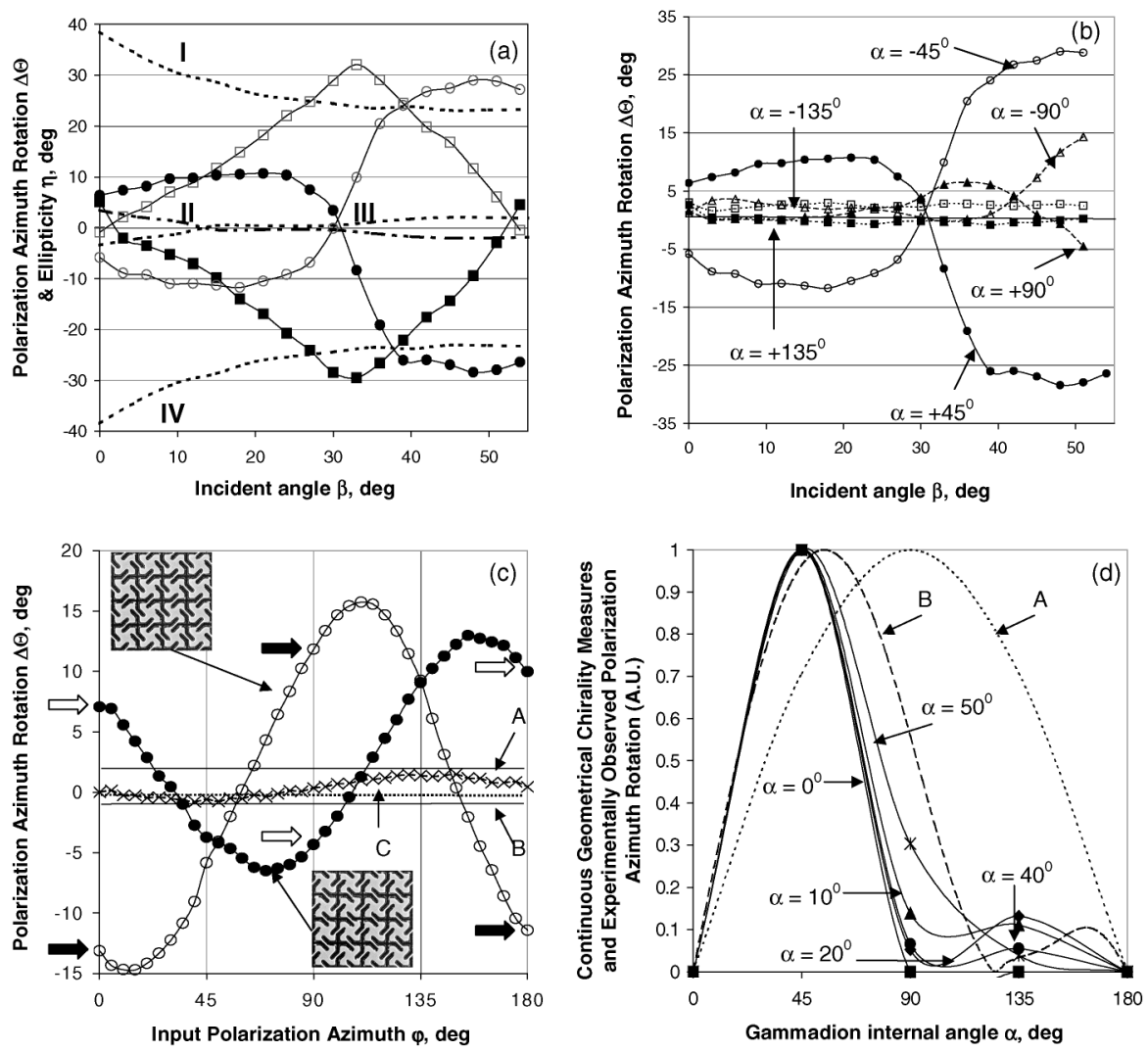

FIG. 2. (a) Polarization azimuth rotation $(\bullet, \bigcirc)$ and change in ellipticity $(\boldsymbol{\square}, \square)$ of light wave diffracted from left-handed $(\bigcirc$, $\square)$ and right-handed $(\boldsymbol{\square}, \mathbf{\square})$ gammadion arrays plotted as functions of the incidence angle. The two enantiomeric forms correspond to $\alpha=-45^{\circ}$ (left handed) and $\alpha=+45^{\circ}$ (right handed). Note the change of the sign of the polarization effects from left- to righthanded structures. For illustration and comparison purposes, theoretical results are presented which show polarization azimuth rotation (lines I and IV) and ellipticity (II and III) for a complementary design of gammadions made of an ideal conductor (I and III: left-handed gammadions; II and IV: right-handed gammadion). (b) Polarization azimuth rotation of light wave diffracted from left-handed $(\bigcirc, \triangle, \square)$ and right-handed $(\boldsymbol{O}, \mathbf{\Delta ,} \mathbf{\square})$ gammadion arrays of different degrees of chirality plotted as functions of the angle of incidence. The different plots correspond to different internal angles $\alpha=-45^{\circ}(\bigcirc), \alpha=-90^{\circ}(\triangle), \alpha=-135^{\circ}(\square)$, $\alpha=+45^{\circ}(\mathbf{O}), \alpha=+90^{\circ}(\mathbf{\Delta}), \alpha=+135^{\circ}(\boldsymbol{\square})$. (c) Polarization azimuth rotation of light wave diffracted from left-handed ( $\bigcirc$ ) and right-handed (O) gammadion arrays and reflected from an unstructured Ti/Au/Ti surface $(\times)$ plotted as functions of the polarization azimuth of the incident wave. The lines $A, B$, and $C$ are the orientation mean values of the rotations for right- and lefthanded gammadions and unstructured metal, respectively. Note that when the unstructured metal shows no polarization change $\left[\varphi=0\right.$ (180), $\left.90^{\circ}\right]$, left- and right-handed gammadion structures show opposite rotations, as indicated by the arrows. (d) Comparison of theoretical values of the continuous chirality measures calculated using the Zabrodsky and Avnir model (curve $A$ ), and using formula (1) (curve $B$ ), with experimentally observed values of azimuth rotation (solid connecting lines have been added by hand to distinguish between gammadions with different angles). In all cases normalized absolute values (magnitudes) are presented.

chirality, constitutes about $12 \%$ of the total chiral effect measured at $\varphi=90^{\circ}$ and $\varphi=0^{\circ}$. Therefore, structural and molecular planar chirality are both seen in our artificial gammadion gratings.

It is possible to establish a continuous measure of planar geometrical chirality, which raises the intriguing question of how this measure is related to the strength of observable chiral optical effects. However, such a relationship depends on the excitations created by light in the structure and will undoubtedly depend as much on the material characteristics of the structure as on its geometry. Nevertheless, analysis of the geometrical chirality may be used as a relatively simple first step in identifying the most promising chiral structures. To illus- trate this, we compare our experimental results with two models of continuous chirality. The first, the Zabrodsky and Avnir model [3], introduces a continuous chirality scale based on the minimal distances that the vertices of a shape must move in order to attain the nearest achiral configuration, which in our case we assumed to be a grid of crosses. The second model, which we introduce here [10], evaluates the chirality of a set of points by summing the individual chirality measures of all possible triangles made from the points. If a particular triangle has sides of length $a_{1}, a_{2}$, and $a_{3}$, the chirality measure for that triangle is calculated by summing the differences in areas for pairs of subtriangles created by bisecting each of the angles in turn. This results in the following 
formula: $\Gamma\left(\mathbf{r}_{1}, \mathbf{r}_{2}, \mathbf{r}_{3}\right)=\left(a_{1}-a_{2}\right)\left(a_{1}+a_{2}\right)^{-1}+\left(a_{2}-a_{3}\right) \times$ $\left(a_{2}+a_{3}\right)^{-1}+\left(a_{3}-a_{1}\right)\left(a_{3}+a_{1}\right)^{-1}$. By summing over all possible combinations of the points on the shape, one will arrive at the following chirality measure $K$ :

$$
\begin{aligned}
K=\frac{1}{6} \iiint \rho\left(\mathbf{r}_{1}\right) \rho\left(\mathbf{r}_{2}\right) \rho\left(\mathbf{r}_{3}\right) \Delta\left(\mathbf{r}_{1}, \mathbf{r}_{2}, \mathbf{r}_{3}\right) \\
\times \Gamma\left(\mathbf{r}_{1}, \mathbf{r}_{2}, \mathbf{r}_{3}\right) d \mathbf{r}_{1} d \mathbf{r}_{2} d \mathbf{r}_{3} .
\end{aligned}
$$

Here $\Delta\left(\mathbf{r}_{1}, \mathbf{r}_{2}, \mathbf{r}_{3}\right)$ is the area of an individual triangle defined by coordinate vectors $\mathbf{r}_{1}, \mathbf{r}_{2}$, and $\mathbf{r}_{3}$ and $\rho(\mathbf{r})$ is the point density function. We find it remarkable that the experimentally observed magnitude of the polarization azimuth rotation shows some correlation with both geometrical chirality measures and particularly strong correlation with that calculated using formula (1) [see Fig. 2(d)].

On the microscopic level the mechanism behind optical manifestations of planar chirality may be understood if one considers an electromagnetic field polarizing a gammadion. If a conductive gammadion interacts with the electric field of the incident wave, directed, for instance, along one of the gammadion legs, the positive and the negative carriers are driven into opposite legs. As the conductive strip is bent in a chiral fashion, charges will be shifted not only along the direction of the field, but also into the bent ends of the legs, thereby inducing a polarization perpendicular to the driving electric field with a sign that depends on the direction of the bend and, therefore, on the chirality of the gammadion. When fields are re-emitted by the chiral currents, a rotation of the polarization state of the scattered field is observed. The diffraction pattern and polarization properties of the diffracted waves are formed by a coherent addition of fields scattered from individual elements in a given direction. We were able to compare our experimental data with values calculated using a numerical model which incorporates this microscopic mechanism [11]. This numerical code was originally developed for microwave application and treats chiral gammadions as thin narrow strips of ideal conductors, an assumption which is not strictly correct in the optical part of the spectrum. Moreover, in our experiments we used gammadion structures etched into a gold film, where the chiral response is likely to be formed by excitation of plasmons in grooves of the structure [12], so they are complementary to gammadions made from strips of conducting metal, as assumed in the calculation. In spite of these obvious discrepancies between the model and the actual experimental situation, we believe that it is instructive to compare experimental data with the results of these calculations, which we present in Fig. 2(a). The model predicts many essential features of the observed polarization effect, namely, opposite signs of the rotation and elliptization for enantiomeric forms, pronounced dependence of the effect on the incident angle, and the characteristic magnitudes of rotation and elliptization.
To summarize, we observed for the first time unambiguous evidence of optical manifestation of chirality in a two-dimensional planar system in the far-field diffraction regime. Possibly the most intriguing feature of planar chirality would be that the sign of chirality reverses if the structure is observed from different sides of the plane and so should be the polarization effect associated with it [5]. If a subwavelength 2D chiral structure would show a polarization effect in the absence of diffraction, its nonreciprocal features should resemble those of the optical Faraday effect of polarization plane rotation in magnetized media and contrasts with conventional optical activity where polarization azimuth rotation caused by three-dimensional chirality does not depend on the direction of propagation. As reversing of the propagation direction is equivalent to the time reversal $T$ operation, polarization azimuth rotation on transmission through a planar chiral structure would be a relatively rare manifestation of a $T$-odd effect in optics. So far, using the 2D chiral structures described above, we have been able to see that for a transmission diffraction experiment at a wavelength of $1.52 \mu \mathrm{m}$ the polarization eigenstates change if the direction of light propagation through the structure is reversed.

The authors acknowledge the support of the Science and Engineering Research Council (U.K.) and fruitful discussions with L. Arnaut, L. Barron, L. Hecht, and R. Greef.

*To whom all correspondence should be addressed. Email address: n.i.zheludev@soton.ac.uk

[1] L. R. Arnaut, J. Electromagn. Waves Appl. 11, 1459 (1997).

[2] P. LeGuennec, J. Math. Phys. (N.Y.) 41, 5954 (2000).

[3] H. Zabrodsky and D. Avnir, J. Am. Chem. Soc. 117, 462 (1995).

[4] M. A. Osipov, B.T. Pickup, M. Fehervari, and D. A. Dunmur, Mol. Phys. 94, 283 (1998).

[5] L. Hecht and L. D. Barron, Chem. Phys. Lett. 225, 525 (1994).

[6] L. R. Arnaut and L. E. Davis, in Proceedings of the International Conference on Electromagnetics in Advanced Applications (Nexus Media, Swanley, U.K., 1995), pp. 381-384.

[7] V. P. Drachev, W. D. Bragg, V. A. Podolskiy, V. P. Safonov, W.T. Kim, Z.C. Ying, R. L. Armstrong, and V.M. Shalaev, J. Opt. Soc. Am. B 18, 1896 (2001).

[8] See http://xahlee.org/Wallpaper_dir/c5_17Wallpaper Groups.html

[9] Yu. P. Svirko and N. I. Zheludev, Polarization of Light in Nonlinear Optics (Wiley, Chichester, 1998), Chap. 3.

[10] Details of the theory will be published elsewhere.

[11] S. L. Prosvirnin, J. Commun. Technol. Electron. 44, 635 (1999).

[12] M. B. Sobnack, W. C. Tan, N. P. Wanstall, T.W. Preist, and J. R. Sambles, Phys. Rev. Lett. 80, 5667 (1998). 